\title{
Switching a magnetic vortex by interlayer coupling in epitaxially grown $\mathrm{Co} / \mathrm{Cu} / \mathrm{Py} / \mathrm{Cu}(001)$ trilayer disks
}

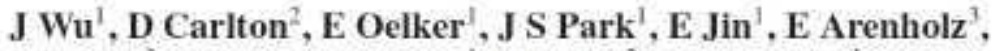 \\ A Scholl ${ }^{3}$, Chanyong Hwang ${ }^{4}, \mathrm{~J}$ Bokor ${ }^{2}$ and Z Q Qiu' \\ ${ }^{1}$ Department of Physics, University of California at Berkeley, Berkeley. CA 94720, USA \\ ${ }^{2}$ Depastment of Electrical Engineering and Computer Sciences, University of Califormia at \\ Berkeley, Berkeley, CA 94720 , USA \\ ${ }^{3}$ Advanced Light Source, Lawreace Berkeley National Lahoratory Berkeley, CA 94720 , USA \\ ${ }^{4}$ Korea Research Institule of Standards and Science, Yuscongt Daejeon 305-340, Korea \\ E-mail: qiue vocrates berheley.edu
}

Received 16 July 2010

Published 3 August 2010

Online at stacks.iop.org/JPhysCM/22/342001

\begin{abstract}
Epitaxial Py/Cu/Co/Cu(001) trilayers were pattemed into micron sized disks and imaged using element-specific photoemission electron microscopy. By varying the $\mathrm{Cu}$ spacer layer thickness: we study how the coupling between the rwo magnetic layers influences the formation of magnetic vortex states. We tind that while the Py and Co disks form magnetic vortex domains when the interlayer coupling is ferromagnetic, the magnetic vortex domains of the Py and Co fisks hreak into anti-parallel aligned multidomains when the interlayer coupling is antiferromagnetic. We explain this result in terms of magnetic flux closure between the Py and Co layers for the antiferromagnetic coupling case.
\end{abstract}

\section{Introduction}

Understanding the formation of various magnetic states in contined geometries is crucial to the development of spintronics technology. The magnetic vortex state in magnetic disks is an important example of magnetic sate formation in nanostructures and has been a recent area of interest [1]. A magnetic vortex state is formed when the spin magnetization directions curt inside a disk to minimize the system's total magnetic energy [2]. While much effort has been devoted to the study of single layer vortex state such as the vortex dynamics [3] and vortex core reversal [4], multilayer structures are less well understood. Recent studies have indicated that vortex states in trilayer disks exhibit many new interesting phenomena due to the magnetic coupling between vortices $[5,6]$. For example, magnetic domain imaging reveals that the magnetization reversal in a trilayer structure involves a coordinated magnetic reversal of each magnetic layer [7]. Magnetic simulations also show that the vortex dynamics in a trilayer structure is highly non-linear, Fevealing nontrivial coupled vortex dynamics [8].

However, most previous work has studied vortex formation in polycrystalline films in which the two disks are strongly ferromagnetically coupled. In the limit of infinitely strong ferromagnetic coupling, the magnetic states in the two magnetic disks will be identical so a trilayer system should have no fundamental difference from a single layer system. On the other hand, layers that are antiferromagnetically coupled are not well studied. To investigate the formation of antifertomagnetically coupled magnetic domain states in these confined structures, we fabricated high quality single crystal trilayer disks in which the interlayer magnetic coupling can be tuned systematically between ferromagnetic coupling $(\mathrm{FC})$ and antiferromagnetic coupling ( $\mathrm{AFC}$ ) by varying the thickness of a nonmagnetic spacer. Using element-specitic X-ray magnetic circular dichrsism (XMCD), we obtained the domain images for each the two coupled ferromagnetic microstructures and unexpectedly find that while FC coupled 


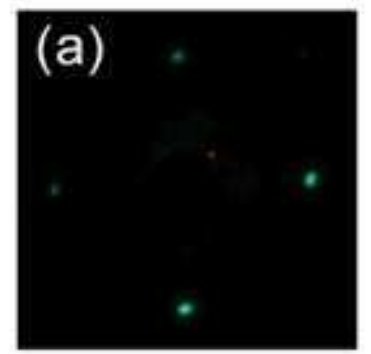

Cu(001)

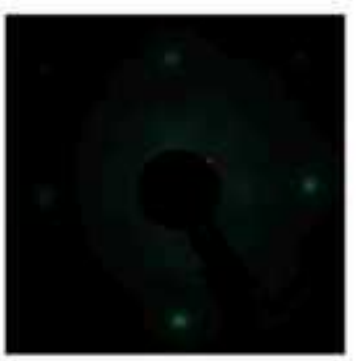

$\mathrm{Co} / \mathrm{Cu} / \mathrm{Py} / \mathrm{Cu}(001)$

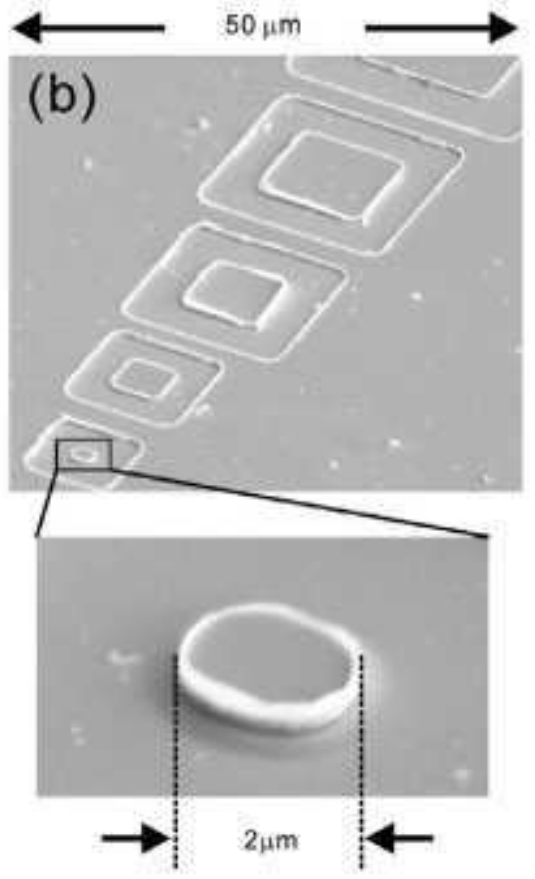

Figure 1. (a) LEED result shows the formution of single crystaltine Co/Cu/Py/Cuif001) films. (b) SEM imuge of F1B fabricated disks from the $\mathrm{Co} / \mathrm{Cu} / \mathrm{Py} / \mathrm{Cu}(001)$.

(This figure is in colour only in the electronic version)

microstructures favor the formation of magnetic vortex states, AFC coupled microstructures do not form vortex states.

\section{Experiment}

The microstructures were fabricated on a $\mathrm{Cu}(001)$ single crystalline substrate, which was cleaned in an ultrahigh vacuum system by cycles of $\mathrm{Ar}$ ion sputtering at $2 \mathrm{keV}$ and annealing at $600^{\circ} \mathrm{C}, \mathrm{Py}, \mathrm{Cu}$, and $\mathrm{Co}$ films were grown epitaxially onto the $\mathrm{Cu}(001)$ substrate at room temperature to form $\mathrm{Co}(30 \mathrm{~A}) / \mathrm{Cu} / \mathrm{Py}(120 \mathrm{~A}) / \mathrm{Cu}(001)$ trilayer structure. The Cu spacer layer was grown into a wedge ( $0-15 \mathrm{ML}$ over $2 \mathrm{~mm}$ ) to facilitite a continuous thiekness variation. It is well known that it magnetic sandwich structure exhibits an oscillatory magnetic interlayer coupling across a spacer layer $|9|$ due to the formation of quantum well states in the spacer layer [10]. Thus the interlayer coupling between the Py and Co layers in our sample should oscillate between ferromagnetic coupling (FC) and antiferromagnetic coupling (AFC) as a function of the Cu spacer liyer thickness.

After capping the trilayer with a $20 \mathrm{~A}$ Cu capping layer to prevent oxidation of the magnetic thin film, the sample was characterized by low energy electron diffraction (LEED). The sharp LEED spots from the trilayers are almost identical to that of the $\mathrm{Cu}(0 \mathrm{OH})$ substrate (figure $\mathrm{I}(\mathrm{a})$ ). indicating that high quality epitaxial single crystalline $\mathrm{Co} / \mathrm{Cu} / \mathrm{Py}$ trilayers are formed on the $\mathrm{Cu}(001)$ substrate. The sample was then taken out of the UHV chamber and patterned using standard photolithography and ion milling. The resulting structure exhibits sharp edges and a complete removal of the trilayers from the designed area for the fabricated disks (figure l(b)).

\section{Results and discussions}

To characterize the sample magnetically, we first performed XMCD measurements on an unpatterned area of the $\mathrm{Co} / \mathrm{Cu} / \mathrm{Py} / \mathrm{Cu}(\mathrm{OO} 1)$ substrate. Photoemission electron microscopy (PEEM) was used to image the Co and Py domains at the PEEM-II beamline of the advanced light source. A circularly polarized $\mathrm{x}$-ray bean is incident at a $60^{\circ}$ angle relative to the sample surface normal direction. By tuning the $\mathrm{x}$-raly energy to the $\mathrm{Co}$ and $\mathrm{Ni} 2 \mathrm{p}$ level absorption edge, we obtained the Co and Py magnetic domain images separately [11]. Figure 2 shows representative PEEM images for different $\mathrm{Cu}$ thicknesses $\left(d_{\mathrm{Cu}}\right)$. At $d_{\mathrm{Cu}}=3.5 \mathrm{ML}$, the $\mathrm{Co}$ and Py magnetic domains have identical domain pattem and contrast, showing that the $\mathrm{Co}_{0}$ and $\mathrm{Py}$ magnetic spins are parallel to each other wirh ferromagnetic interlayer coupling between the $\mathrm{Co}$ and $\mathrm{Py}$. At $d_{\mathrm{CI}}=6.5 \mathrm{ML}$, however, the $\mathrm{Co}$ and $\mathrm{Py}$ magnetic domains have identical domain pattern but have opposite magnetization contrasts, showing that the Co and Py magnetic spins are anti-parallel to each other with antiferromagnetic interlayer coupling. The above result was further confirmed by magnetic hysteresis loop measurements obtained using a standard magneto-optic Kert effect (MOKE) setup. At $d_{\mathrm{Cu}}=3.5 \mathrm{ML}$, the hysteresis loop exhibits square shape with a full remanence because the $C o$ and $P y$ switch together with the magnetic field (figure 2). At $d_{\mathrm{Cu}}=6.5 \mathrm{ML}$, 


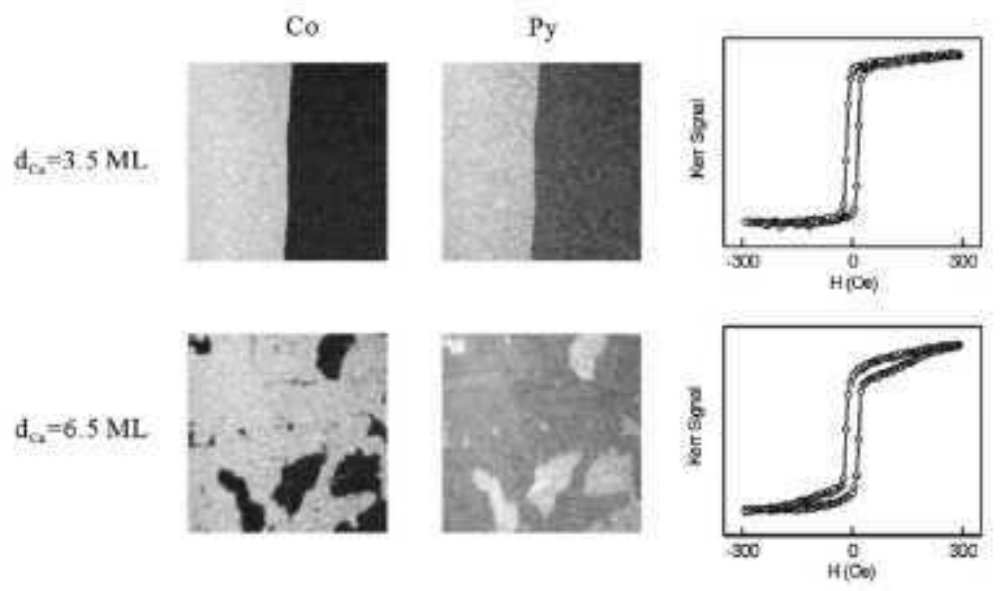

Figure 2. PEEM domain images (left) and MOKE hysteresis loops (right) show that the Co ind Py layers are ferromagnetically coupled across $3.5 \mathrm{ML}$ Cu spacer layer and antiferromagnetically coupled across $6.5 \mathrm{ML}$ Cu spacer layer.

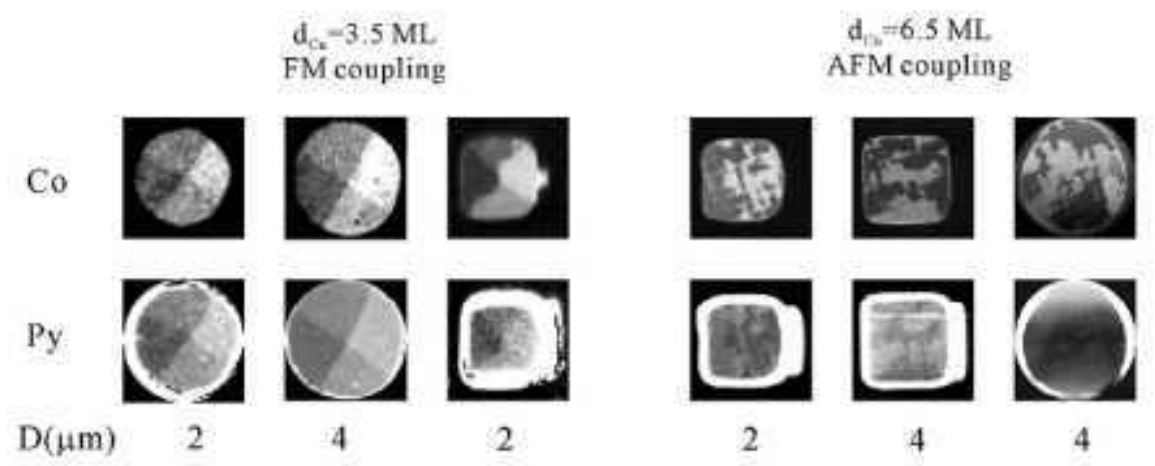

Figure 3. The $\mathrm{Co} / \mathrm{Cu} / \mathrm{Py} / \mathrm{Cu} / \mathrm{O0} 1$ ) trilayer disks exhibits magnetic vottex state for the ferromagnetic interiayer coupling case $\left(d_{\mathrm{C}}=3.5 \mathrm{ML}\right.$ ). but multimagnetic domains for the antiferromagnetically coupled case $\left(d_{\mathrm{Ce}}=6.5 \mathrm{ML}\right.$ ).

the magnetization does not have a full remanence due to the anti-parallel alignment of the $\mathrm{Co}_{\mathrm{O}}$ and Py layers and is saturated at a much higher magnetic field compared to the $d_{\mathrm{CII}}=3.5 \mathrm{ML}$ measurement. This result is consistent with antiferromagnetic interliyer coupling between the $\mathrm{Co}$ and Py layers. Together. the PEEM and MOKE result confirms the ferromagnetic and antiferromagnetic couplings between the $\mathrm{Co}$ and Py layers across $d_{\mathrm{Cu}}=3.5$ and $6.5 \mathrm{ML}$, respectively.

Element-specific domain images were next obtained on the patterned regions of the substrate at roum tempetature. In the $d_{\mathrm{C} I 1}=3.5 \mathrm{ML}$ region of the sample, where the Co and Py are eoupled ferromagnetically, we observed at magnetic vortex state clearly (tigure 3). Moreover, the vortex state exists in both circular and square structures of diameter/length 2 and $4 \mu \mathrm{m}$. Previous work studying ferromagnetic coupling in pattemed polycrystalline thin films has shown that the magnetization vector curls around the center of the disk continuously. However. our results show that the magnetization vector is along the $[ \pm 1, \pm 1,0]$ axes and switches its direction by forming is domain wall. This is due to the four-fold magnetocrystalline anisotropy of the $\mathrm{Co}$ film, which results in an easy magnetization axis in the $[ \pm 1, \pm 1,0]$ axes. In a polycrystalline disk the magnetocrystalline anisotropy direction is averaged out due to the random alignment of the crystal grains. The Py layer lacks the four-fold magnetocrystalline anisotropy that the $\mathrm{Co}$ layer has, but we find the yortex structure to be oriented identically to the Co disk. An isolated Py disk should have a continuous change in the magnetization vector in analogy with a polyerystalline layer, but instead the domain wall pattern of the Co layer is imprinted into the Py disk as a result of the coupling between the rwo lisyers. Because Py has a weaker moment than $\mathrm{Co}$, applying an external magnetic field should affect the two vortices differently. The application of an extemal field is problematic in PEEM due to the interaction of the magnetic field with the emitted electrons used to obtain the image, but work is underway to study this effect using $\mathrm{x}$ ray transmission microscopy, which is more compatible with externally applied fields.

For the case of $d_{\mathrm{CI}}=6.5 \mathrm{ML}$, where the Co and Py layers are antiferromagnetically coupled, we do not observe any vortex states. The sample was demagnetized using standard AC demagnetization techniques, but we always obtained either single domain or multidomain states, never a vortex state (figure 3 ). But regardless the domain shape, we always have the $\mathrm{Co}_{\mathrm{B}}$ and $\mathrm{Py}$ magnetizations anti-parallel to each other. This result shows that the single and multidomain stites are almost energetically degenerated, but have a lower 
energy than the vortex state under this condition. The absence of the vortex state is tot due to the absence of interlayer magnetic coupling because the $C_{0}$ and $P y$ domains are always antiferromagnetically coupled no matter what the multidomain partern looks like after the demagnetization. It is also not likely due to damage of the sample during patterning because the same patterning techniques were used for both the ferromagnetically and antiferromagnetically coupled structures and all of different the patterned structures are from the same substrate. The absence of the vortex in the antiferromagnetically coupled microstructures also doesa't depend on their size and shape. Both square and circular disks at 2 and $4 \mathrm{rm}$ diameters fail to exhibit vortex state for the antiferromagnetic interlayer coupling. The small size of the multidomains suggests that the vortex state should be absent at even smaller sizes. Therefore, we conclude that the vortex state is not the ground state for antiferromagnetically coupled trilayer disks.

The magnetic ground state of a confined structure forms us a result of the interplay between the film's magnetostatic energy and exchange interactions. The lack of vortex states in antiferromagnetically coupled layers can be explained as a consequence of the minimization of the total energy due to these interactions. For a single ferromagnetic disk, the strong exchange interaction makes it difficult to change the spin direction in the surface normal direction so that the only way to minimize the magnetic field lenkage is by curling the spin direction within the tilm plane, i.e., by forming a magnetic vortex within the disk. In an antiferromagnetically coupled triliyer disk. howeyer, the magnetic field leaking out of one ferromagnetic disk can be converged back into the second ferromagnetic disk because of the anti-parallel spin alignment of the two ferromagnetic layers. Therefore the formation of a vortex state becomes less favored in an antiferromagnetically coupled trilayer disk. The above explanation could account for a certain thickness range but won't work for any thickness ratio of the two ferromagnetic layers. The reason is that the magnetic flux out of a single domain disk is proportional to the product of the magnetization and the film thickness so that the magnetic flux out of one ferromagnetic disk in an inriferromagnetically coupled trilayer disk cannot be totally balanced out by the second ferromagnetic layer. More theoretical work is needed to quantitatively explain our result. however.

\section{Summary}

We studied antiferromagnetic and ferromagnetic coupling in single crystalline $\mathrm{Co} / \mathrm{Cw} / \mathrm{Py} / \mathrm{Cu}(001)$ trilayer disks, We find that while the ferromagnetically coupled disks form magnetic vortex state. the vortex state is absent in the antiferromagnetically coupled disks.

\section{Acknowledgments}

This work was supponted by National Science Foundation DMR-0803305. US Department of Energy DE-AC0205CH11231. KICOS through Global Research Laboratory project. Chinese Education Department, and Western Institute of Nancelectronics (WIN).

\section{References}

[1] Shinjo T. Okuno T. Hussdorf R. Shigeto K and Ono T. 2000 Sereace $289030-2$

12] Wachowiak A. Wiebe I, Bode M, Pietzsch O. Morgenstern M and Wiesendanger R 2002 Scichee $298577-80$

13] Choe S. B. Actemam Y. Scholl A, Bater A. Doran A. Stohr J and Padmore H A 2004 Scrence 304 420-2

(4) Waeyenberge B V et al 2006 Nanure 444 $461-4$

15] Castatio F J. Morecroft D, Jung W and Ross C A 2005 Phrs. Rei, Lent. 95137201

[6] Vavassori P. Bonanni V. Busato A. Bisero D, Gubbiotti G. Adeycye A O. Goolatip S, Singh N. Spezzani C and Sacchi M 2008 J. Phys. D: Appl. Phys. 41134014

17) Huang L. Schotield M A and Zhi Y 2009 Appl Pfrys. Leth. 9504250 l

[8] Jun S H. Shim J H. Oh S K. Yu S C. Kim D H. Mesler B and Fischer P 2009) Appl Plys, Len, 95 142509-11

19] Parkin S S P. More N and Roche K P 1990 Ptoys. Res: Letr. $642304-7$

[10] Kawakami R K. Rotenberg E. Escorcia-Apuricio E J, Choi H J. Wolfe J H. Smith N V and Qiu Z Q 1999 Phos. Rex. Lett. $82+098-101$

111] Wu YZ, Schmid A K and Qiu ZQ 20006 Phys. Rev Lett. 97217205 